\title{
Note from the Publisher
}

I regret to announce that this will be the last issue of NanoBiotechnology. Launched in 2005, NanoBiotechnology started strong, but manuscript submissions have declined in recent years. The number of submissions is no longer adequate to maintain the journal and we have decided to cease publication.

We are grateful to the time, effort and expertise of our Editor-in-Chief, Dr. Tuan Vo-Dinh. We also thank the Editorial Board for their years of service to the journal. These editors and the reviewers who spent time ensuring only quality manuscripts were accepted have been a great help to NanoBiotechnology.

All articles published in the journal will remain online. They will continue to be read and provide an on-going legacy for the journal.

Michael D. Weston

Senior Editor

Biomedical Engineering

Springer Science + Business Media 